\title{
Sensing Motion by Monitoring and Detection of Moving Objects
}

\author{
Gaganpreet \\ Student \\ Department of C.S.E \\ A.I.E.T Faridkot, Punjab
}

\author{
Arshdeep Singh \\ Asst. Professor \\ Department of C.S.E \\ A.I.E.T Faridkot, Punjab
}

\begin{abstract}
Detection of movement of objects is very important in various areas. In this paper we present various techniques related to motion detection of moving objects. Existing methods for moving object detection are mainly the frame subtraction method, the background subtraction method and the optical flow method. The aim is to develop mathematical models, algorithms and technologies to build a machine with vision capabilities as advanced at least as human eyesight.
\end{abstract}

\section{Keywords}

Motion detection, Frame subtraction, Background subtraction, Optical flow method.

\section{INTRODUCTION}

Movement is the act or process of moving a object or person. The movement system provides a continual care and the movement of persons from the site. A gradient-based block matching algorithm[1] to estimate the global motion vectors. So, three steps searching (TSS) algorithm and the sum of absolute difference (SAD) algorithm are used. Then, those algorithms can compensate the motion background as static background through registration difference (RS). The moving object in the inter-frame difference will occur as a high value, while background will represent a lower value respectively. To achieve an accurate object extraction result, the higherorder statistics (HOS) algorithm is used to discriminate backgrounds and moving objects. The higher-order statistics (HOS) algorithm has the good attribution in extraction of the non-Gaussian signal against the Gaussian noise, the method based on four moments of difference image for extracting moving objects is used. Detection of changes from a stationary camera is simpler because it involves fewer estimation procedures steps. Initial approaches in this field involve discreet cosine transform (DCT) coefficients domain approach of video sequences which presents a medical application of patient monitoring via video recording, which is commonly used in hospitals for clinical diagnosis [2]. Automatic detection of the movements of patient in the video may facilitate this application in certain significant aspects. Those certain significant aspects are: First, Detecting unusual changes and abnormal behavior in a scene so that an alarm can be whistled in case of an unusual event. Second, controlling the camera automatically to present a neat and clean view of the object and organizing the video data according to the movement activity of the patient. This approach helps in testing that whether a pixel or a region between two images is significantly different or not [2]. In most compression methods, images are decomposed into blocks containing pixels which are then transformed into DCT coefficients.

\section{BACKGROUND SUBTRACTION METHOD (BSM)}

This is widely used method to detect moving objects[3][4]. Basic idea is to detect the moving object from the difference of an existing frame and a reference frame. The accuracy of the results depend on the image initialization and updating the image (see figure 1).

\subsection{Background Image Initialization}

There are number of methods used for background initialization. For example using the first frame directly or taking the average pixel brightness of initial frames and many more. The most commonly used method is average method[4]. To remove the errors caused by shadow the median method is preferred. Expression is as follows :

$\operatorname{Binit}(\mathrm{x}, \mathrm{y})=$ median $\mathrm{fk}(\mathrm{x}, \mathrm{y}) \mathrm{k}=1,2 \ldots . \mathrm{n}$

Where Binit is initial background, $\mathrm{n}$ is total no of frame selected.

\subsection{Moving Object Mining}

To recognize the moving object the most common technique is Background Subtraction. In this technique the subtraction of an image without an object and an image with an object is done. The difference gives a set of pixels that represents the moving object [5].

Subtract the background image $\mathrm{B}(\mathrm{x}, \mathrm{y})$ from the $\mathrm{Fk}(\mathrm{x}, \mathrm{y})$. if the pixel difference is greater than the set threshold than the pixel appear in the moving object.

Algorithm is described in the following steps (see figure 1):

1. Input the Sequences of Video Frames

2. Separate the frames obtained in step (1).

3. Perform the operation of Image Sequence.

4. Perform Separation of Image Sequence in Current Frame Image and Background Frame Image

5. Perform Background subtraction

6. Detection of Moving Object is performed by obtaining the information from step 4 and 5 .

7. Background updating

8. Perform Noise Removal Operation on extracted frames

9. Perform Shape Analysis in the current frame

10. Display the result.

11. End. 


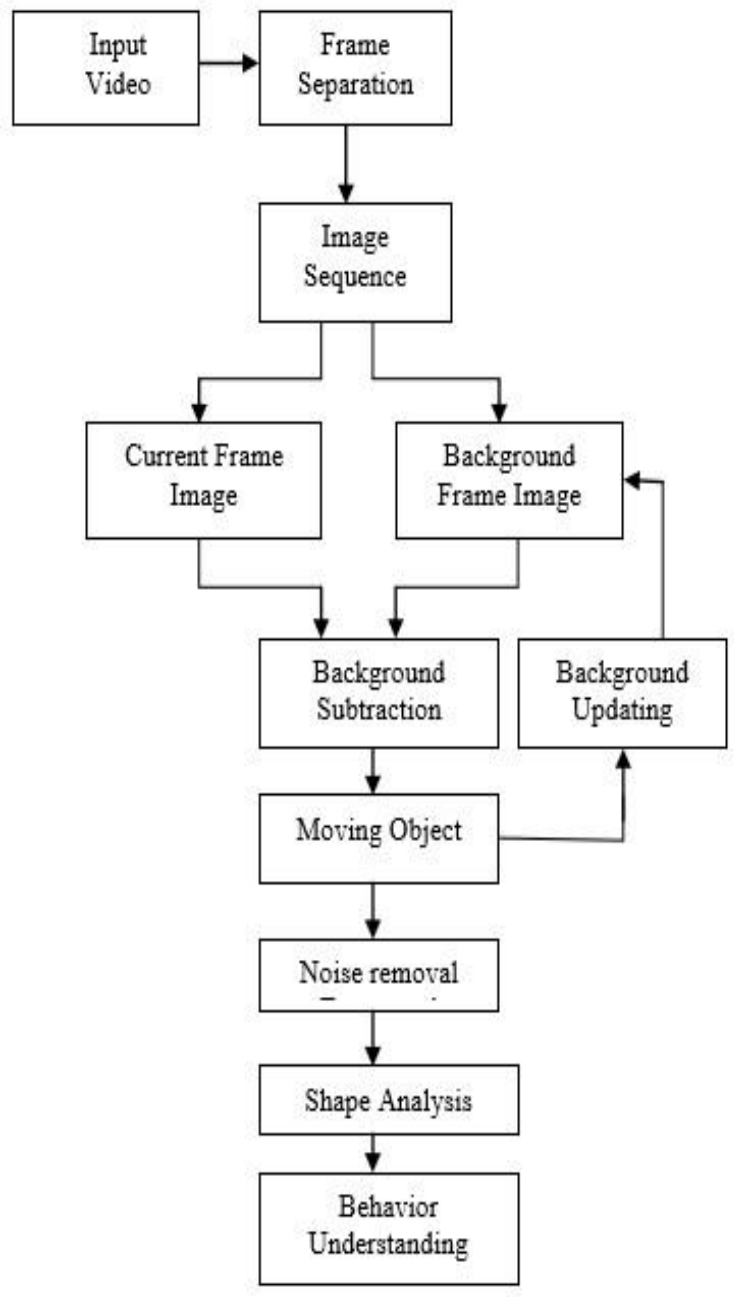

Fig 1: Background Subtraction

\section{CONSECUTIVE FRAME SUBTRACTION METHOD}

In this method the calculation of difference between the pixels in consecutive frames of a video sequence is done [9]. This indicates the motion (see figure 2).

Algorithm is described as in the following steps:
1. First we attach a stationary High quality camera with the system. Then we select the portion of which we want to capture movement by using GUI Buttons.

2. After this we use GUI start monitoring button for start the movement capturing.

3. The stationary camera captures the real time video by which the system can store the current frame and last frame of video.

4. Then the algorithm divides the each frame in to pixel by pixel and find the RGB value of each pixel.

5. Then convert the value of pixels into array of rows and columns.

6. After this subtract the RGB value of each pixel of last frame from the current frame.

7. Then after subtraction of RGB values of two frame, it calculates the absolute value of each pixel.

8. By using absolute value, if the result is in the negative integer it converts into the positive integer.

9. Then convert the RGB value in to black \& white which shows only two values of each block i.e. 0 and 1 .

10. After this combine the binary value of each block to make a new image and open it into black \& white area in which the changes show the white i.e. $1 \&$ unchanged portion shows the black i.e. 0 .

11. Then calculate the percentage of changes of the selected portion, by using the changed area w.r.t whole selected area.

12. Store the current frame into previous frame.

13. At the end the graph will show the percentage of movement w.r.t time in seconds.

Repeat steps from 3 to 13 until there is last frame. 


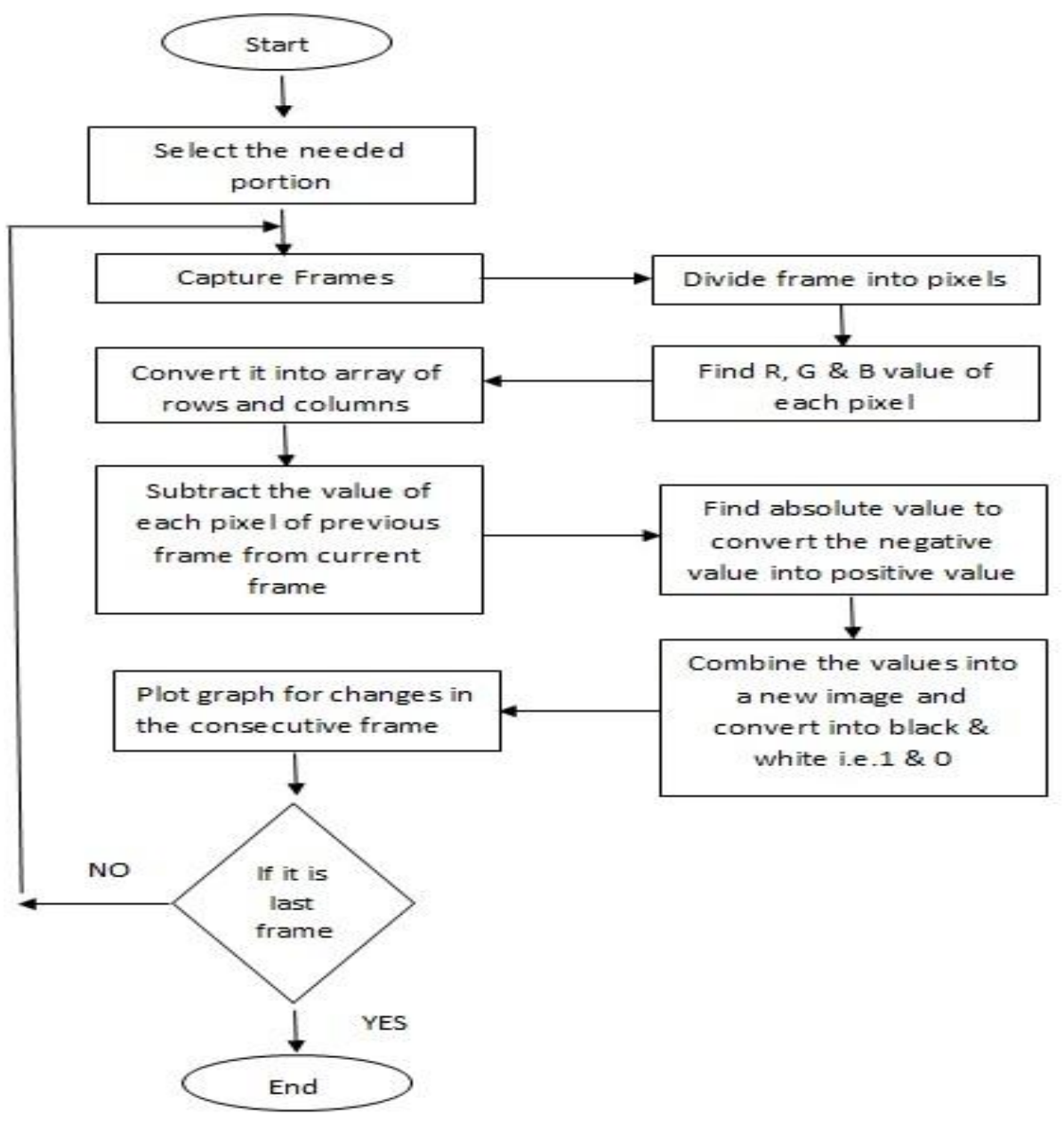

Fig 2: Frame Subtraction

\section{OPTICAL FLOW METHOD}

An image flow field is 3-D velocity vector of objects, projected onto the image plane and the movement of pixels in an image is termed as optical flow field. It is the motion calculated between two image frames taken at interval $t$ at every pixel position[11]. The mathematical representation is:

$$
I(x . y . t)=I(x+\Delta x, y+\Delta y, t+\Delta t)
$$

where $f(x, y, t)$ is the intensity of the image at position $(x, y)$ and at time $t$, and $\Delta x, \Delta y$ is the change in position and $\Delta t$ is the change in time. After applying Tayler series expansion:

$I(x+\Delta x, y+\Delta y, t+\Delta t)=I(x \cdot y \cdot t)+\frac{\partial I}{\partial x} \Delta x+\frac{\partial I}{\partial y} \Delta y+$

$\frac{\partial I}{\partial t} \Delta t+$ h.o.t

where $\partial \mathrm{I} / \partial \mathrm{x}, \partial \mathrm{I} / \partial \mathrm{y}, \partial \mathrm{I} / \partial \mathrm{t}$ are the partial derivatives of the image function in the $\mathrm{x}, \mathrm{y}$, and $\mathrm{t}$ dimensions and h.o.t represents higher order terms.

If we substitute equation 1 into 2 , the result is:

$\frac{\partial I}{\partial x} \Delta x+\frac{\partial I}{\partial y} \Delta y+\frac{\partial I}{\partial t} \Delta t=0$

Or

$\frac{\partial I}{\partial x} \frac{\Delta x}{\Delta t}+\frac{\partial I}{\partial y} \frac{\Delta y}{\Delta t}+\frac{\partial I}{\partial t} \frac{\Delta t}{\Delta t}=0$
Or

$\frac{\partial I}{\partial x} V_{x}+\frac{\partial I}{\partial y} V_{y}+\frac{\partial I}{\partial t}=0$

where $\mathrm{Vx}$ and $\mathrm{Vy}$ are the $\mathrm{x}$ and $\mathrm{y}$ components of the velocity or optical flow of $\mathrm{I}(\mathrm{x}, \mathrm{y}, \mathrm{t})$.

$I_{x} V_{x}+I_{y} V_{y}=-I_{t}$
$\left[\begin{array}{ll}I_{x} & I_{y}\end{array}\right]\left[\begin{array}{l}V_{x} \\ V_{y}\end{array}\right]=-I_{t}$

\subsection{Lucas-Kanade Method}

This is a popular method that involves the differential technique[11][12]. This method involves solving for the optical flow vector by assuming that the vector will be similar to a small neighbourhood surrounding the pixel. Assuming that the flow $(\mathrm{Vx}, \mathrm{Vy})$ is a constant in a small window of size $m \times m$ where $m>1$ and numbering the pixels from 1 to $n$, there are number of equation:

$$
\begin{aligned}
& I_{x_{1}} V_{x}+I_{y_{1}} V_{y}=-I_{t_{1}} \\
& I_{x_{2}} V_{x}+I_{y_{2}} V_{y}=-I_{t_{2}} \\
& \vdots \\
& I_{x_{3}} V_{x}+I_{y_{3}} V_{y}=-I_{t_{3}}
\end{aligned}
$$


In Matrix form,

$\left[\begin{array}{cc}I_{x_{1}} & I_{y_{1}} \\ I_{x_{2}} & I_{y_{2}} \\ \vdots & \vdots \\ I_{x_{n}} & I_{y_{n}}\end{array}\right]\left[\begin{array}{l}V_{x} \\ V_{y}\end{array}\right]=\left[\begin{array}{l}-I_{t_{1}} \\ -I_{t_{2}} \\ \vdots \\ -I_{t_{n}}\end{array}\right]$

$A \vec{v}=-b$

Since this is an over-determined system of equations, so least square method is used to solve the:

$A^{T} A \vec{v}=A^{T}(-b)$

$\vec{v}=\left(A^{T} A\right)^{-1} A^{T}(-b)$

Now, using summation from $i=1$ to $\mathrm{n}$ we have:

$\left[\begin{array}{l}V_{x} \\ V_{y}\end{array}\right]=\left[\begin{array}{cc}\sum I_{x_{i}}^{2} & \sum I_{x_{i}} I_{y_{i}} \\ \sum I_{x_{i}} I_{y_{i}} & \sum I_{y_{i}}^{2}\end{array}\right]^{-1}\left[\begin{array}{l}-\sum I_{x_{i}} I_{t_{i}} \\ -\sum I_{y_{i}} I_{y_{i}}\end{array}\right]$

This method is not suitable when inner parts of large homogenous areas show little motion. The advantage of Lucas-Kanade method its accuracy and robustness of detection in presence of noise.

\section{CONCLUSION}

We have presented a review of popular three Motion Sensing Techniques used in monitoring and detection of moving objects. Extraction of information can provide us object classification and defining the difference between humans, vehicles or animals. The main application is to track the moving objects. The future scope is an infrastructure for the monitoring devices to push their data into, for example a server with a database. It can also be identified in such a way that the monitoring device stores all the data and applications needing data connected directly to the monitoring device.

\section{REFERENCES}

[1] Richard J. Radke, Srinivas Andra et al 2004. Image Change Detection Algorithms: A Systematic survey.

[2] Qiang Liu, Robert J. Sclabassi et al 2005.A DCTDomain Approach to image change detection and its application to patient video monitoring . MultiMedia Signal Processing Workshop, IEEE.

[3] Lijing Zhang ,Yingli Liang 2010. Motion human detection based on background Subtraction. Second International Workshop on Education Technology and Computer Science,IEEE Computer Science IEEE.

[4] Xiaofei Ji, Honghai Liu, 2010. Advances in ViewInvariant Human Motion Analysis:A Review in IEEE Trans.on Systems,Man, Cybernetics,vol.40,no.1.

[5] Hazi Wang and David suter, "Background Subtraction Based on a Robust Consensus Method ," Monash University, Clayton Vic. 3800, Australia.

[6] K. S. Tan, R. Saatchi et al 2010, Real-Time Vision Based Respiration Monitoring System, IEEE.

[7] Yuki Fujimori, Yoshiyuki Ohmura et al 2009. Wearable Motion Capture Suit with Full-body Tactile Sensors, IEEE.

[8] Mohamed F. Abdelkader et al 2006, Integrated Motion Detection and Tracking for Visual Surveillance, IEEE.

[9] Ching Yee Yong, Rubita Sudirman, Kim Mey Chew 2011.Motion Detection and Analysis with Four Different Detectors, Third International Conference on Computational Intelligence, Modelling \& Simulation, IEEE.

[10] Suwich Tirakoat 2011, Optimized Motion Capture System For Full Body Human Motion Capturing Case Study of Educational Institution and Small Animation Production, IEEE.

[11] Francesco Cardile, Giancarlo Iannizzotto et al2010, A Vision Based System for Elderly Patients Monitoring", IEEE.

[11] Nan Lu, Jihong Wang, Q.H. Wu and Li Yang 2008. An Improved Motion Detection Method for Real-Time Surveillance, IAENG International Journal of Computer Science.

[12] B.D. Lucas and T. Kanade, An iterative image registration technique with an application to stereo vision, Proc. 7th International Joint Conference on Artificial 